\title{
Elaboração de um programa para dimensionamento de lajes retangulares de concreto armado submetidas a um carregamento uniforme, com bordos apoiados, engastados ou livres, conforme a NBR 6118:2014
}

Elaboration of a program for the dimensioning of rectangular reinforced concrete slabs subjected to a uniform loading, with edges supported, set or free, according to NBR 6118:2014

\author{
L. A. Costa*; C. H. Viegas; M. W. Moura; M. V. Real \\ Escola de Engenharia/FURG, Universidade Federal do Rio Grande, 96203-900, Rio Grande-RS, Brasil
}

*leidiane.amorim@live.com

\begin{abstract}
Ao longo dos anos, estamos vivenciando uma revolução na área da informática, onde o aumento da capacidade de armazenamento de dados e o desenvolvimento de sistemas computacionais são exemplos bons dessa evolução. Porém, o custo elevado de softwares desenvolvidos para o dimensionamento de estruturas de concreto armado, acarreta no distanciamento deles com os estudantes do curso de engenharia civil e a falta de vivência com os mesmos. Desta forma, o presente trabalho tem como objetivo automatizar e sistematizar através de uma rotina computacional, o dimensionamento de lajes maciças em concreto armado, em formato retangular, submetidas a um carregamento uniformemente distribuídos, podendo estarem apoiadas em bordos apoiados, engastados ou livres, de acordo com o proposto pela norma NBR 6118:2014. Para isto, se utilizou a linguagem de programação Visual Basic, desenvolvida para compilar aplicações orientadas a objeto, possibilitando a utilização do software em ambiente Windows através de uma interface gráfica muito prática e acessível. A partir dos dados de entrada introduzidos pelo usuário, o programa executa o dimensionamento de lajes usuais em edifícios de concreto armado com as características descritas.
\end{abstract}

Palavras-chave: concreto-armado, lajes, algoritmos

Over the years, we are experiencing a revolution in the area of computing, where increasing data storage capacity and developing computer systems are good examples of this evolution. However, the high cost of software developed for the dimensioning of reinforced concrete structures entails their distancing with students of the civil engineering course and the lack of experience with them. In this way, the present work aims to automate and systematize through a computational routine, the dimensioning of solid slabs in reinforced concrete, in a rectangular format, subjected to a uniformly distributed load, and can be supported on supported, set or free edges of according to the one proposed by the norm NBR 6118:2014. For this, we used the Visual Basic programming language, developed to compile object-oriented applications, allowing the use of the software in the Windows environment through a very practical and accessible graphical interface. From the input data entered by the user, the program executes the sizing of usual slabs in reinforced concrete buildings with the characteristics described.

Keywords: concrete-reinforced, slabs, algorithms

\section{INTRODUÇÃO}

Nas últimas décadas, temos vivenciado um avanço considerável no que diz respeito a evolução dos sistemas computacionais. Desta forma, a informática está cada vez mais aperfeiçoando a Engenharia de Estruturas. Com isso, fica evidente a importância de ensinar e preparar os alunos do curso de Engenharia Civil desenvolver, manipular e analisar estruturas de concreto armado com a utilização de softwares destinados para isso [1].

No entanto, o custo elevado dos softwares desenvolvidos com o objetivo de dimensionar estruturas de concreto armado acaba por muitas vezes distanciar os alunos de Engenharia civil dos mesmos.

Desta forma, o objetivo deste trabalho é desenvolver através de uma rotina computacional, um programa capaz de dimensionar lajes maciças de concreto armado, submetidas a um carregamento 
uniformemente distribuído, com a opção para bordos apoiados, engastados e livres, através da norma NBR 6118:2014 [2].

Como ferramenta computacional, foi utilizado o software Visual Basic, desenvolvido para compilar aplicações orientadas a objeto, possibilitando a utilização do software em ambiente Windows através de uma interface gráfica muito prática e acessível. A partir da eficácia do programa, ele poderá ser distribuído para os alunos de Engenharia Civil estudarem tanto a programação desenvolvida, como a utilização do mesmo.

\section{REFERENCIAL TEÓRICO}

Segundo Araújo (2014a) [3], os elementos de piso têm como função principal a distribuição das ações verticais de utilização para as vigas. Nos projetos de concreto armado esses elementos são denominados como Lajes, onde se apresentam na forma plana com a responsabilidade de receber uma boa parte das cargas de uma construção.

Ainda, as lajes de concreto armado podem ser chamadas de placas, a NBR 6118:2014 [2], apresenta o conceito como sendo "Elementos de superfície plana sujeitos principalmente a ações normais ao seu plano".

\subsection{CLASSIFICAÇÃO DAS LAJES}

A classificação das lajes pode ser atribuída segundo a forma de armar, podendo ser dimensionadas para serem armadas em uma única direção (Figura 1) ou em duas direções (Figura 2). Quanto ao seu tipo de apoio e quanto à sua natureza, elas podem ser classificadas como laje maciça, pré-fabricada, nervurada, entre outras.

A possibilidade de armar uma laje em duas direções vem da relação entre o vão maior e o vão menor ser inferior a 2, caso contrário deverá ser dimensionado em uma única direção apenas contendo uma armadura de distribuição na direção de maior vão [3].

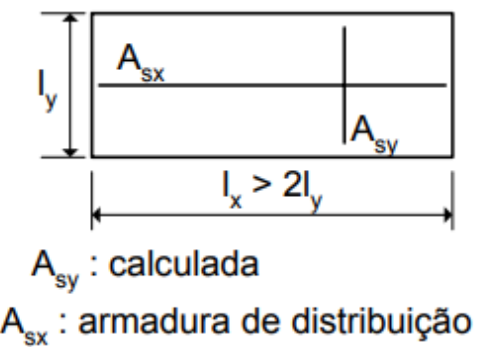

Figura 1: Laje armada em uma direção. Fonte: Araújo (2014) [3].

Segundo Araújo (2014a) [3], quando as lajes forem armadas em cruz (duas direções), como apresentada na Figura 2, os Momentos Fletores das duas direções são importantes e devem ser calculados. Para cada um deles, deve-se realizar o dimensionamento e dispor as armaduras nas direções correspondentes.

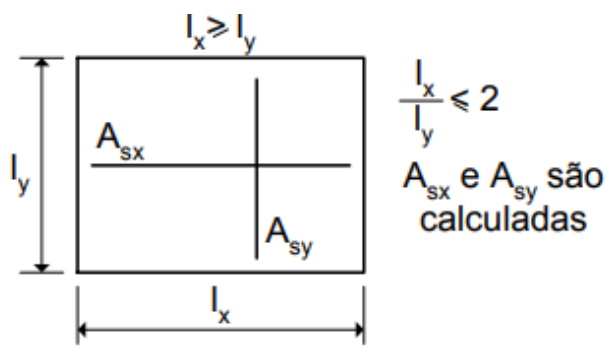

Figura 2: Laje armada em cruz. Fonte: Araújo (2014a) [3]. 


\subsection{CONDIÇÕES DE CONTORNO}

Para calcular nas lajes, os esforços solicitantes e as deformações são necessário determinar suas respectivas vinculações. Por esse motivo são adotadas simplificações, onde se admite que a laje é perfeitamente engastada ou simplesmente apoiada em seu bordo.

Para essas simplificações, usualmente são utilizadas tabelas para cálculo dos esforços solicitantes nas lajes, baseadas na teoria da elasticidade, onde os tipos de apoio utilizados são o apoio simples, a borda livre e o engaste perfeito. Se for analisado pelo que acontece na realidade, é muito difícil a estrutura se apresentar exatamente com o comportamento de apoio simples ou de engaste perfeito. Cunha e Souza (1998) [4] salientam que o erro neste tipo de caso não supera os $10 \%$.

De forma a se identificar o tipo de apoio, tradicionalmente é utilizada uma convenção, apresentados na figura 3 .

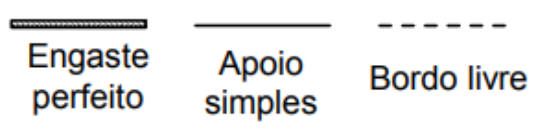

Figura 3: Convenção para as condições de apoio. Fonte: Araújo (2014a) [3].

Segundo Cunha \& Souza (1998) [4], na maioria dos casos a rigidez das vigas de concreto armado à torção é relativamente pequena. Desta forma, se a viga não apresentar uma armadura que resista estes esforços, o concreto fissura, causando deformação e se comportando de acordo com a concepção de apoio simples.

\subsection{DIMENSIONAMENTO AO ESFORÇO DE FLEXÃO SIMPLES}

Algumas hipóteses serão adotadas no dimensionamento das seções transversais em concreto armado sujeitas a flexão simples, baseando-se na NBR 6118:2014 [2] e Araújo (2014b) [5], sendo a hipótese das seções planas, a conjuntura de aderência perfeita entre as barras de aço e o concreto, desprezando a parcela de contribuição de resistência a tração oferecida pela seção tracionada do concreto.

De acordo com a norma NBR 6118:2014 [2], o diagrama tensão-deformação do concreto apresentado na Figura 4 é conhecido como diagrama parábola-retângulo.

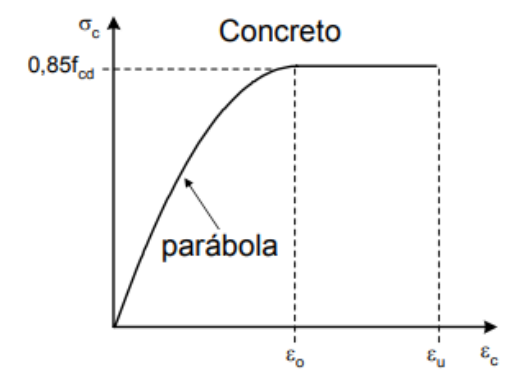

Figura 4: Diagrama parábola-retângulo. Fonte: Araújo (2014a) [3].

O diagrama é composto pela deformação e tensão de compressão no concreto, onde $f_{c d}$ é a resistência à compressão de cálculo do concreto dada pela Equação 1.

$$
f_{c d}=f_{c k} / 1,4
$$

$\mathrm{O}$ valor de $f_{c k}$ é referente à resistência característica a compressão e o valor de 1,4 corresponde a um coeficiente parcial de segurança. As deformações são variáveis conforme o $f_{c k}$, porém, no projeto estrutural é comum assumir $\varepsilon_{o}=0,002$ como valores médios para a compressão simples e $\varepsilon_{u}=0,0035$ para a flexão simples ou composta. 
Pode-se adotar o diagrama tensão-deformação para representar os aços idealizado e sugerido pela NBR 6118:2014 [2], mostrado na Figura 5. O referido diagrama é utilizado para compressão e tração.

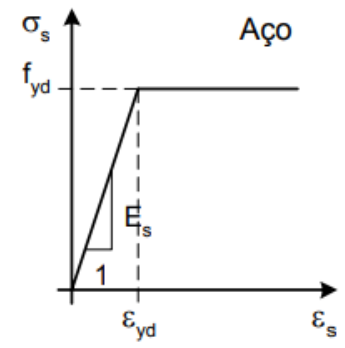

Figura 5: Diagrama tensão-deformação. Fonte: Araújo (2014 a) [3].

O diagrama é composto pela deformação e tensão nas barras de aço, onde $f_{y d}$ é a tensão de escoamento característica do aço dada pela Equação 2.

$$
f_{y d}=f_{y k} / 1,15
$$

$\mathrm{O}$ valor de $f_{y k}$ é referente à tensão de escoamento característica do aço, onde valor de 1,15 representa um coeficiente de segurança. Por deformação plástica excessiva do aço adota-se o valor de $\varepsilon_{s}=0,01$.

A deformação de escoamento de cálculo, $\varepsilon_{y d}$, é dada pela Equação 3.

$$
\varepsilon_{y d}=f_{y d} / E_{s}
$$

Onde o módulo de elasticidade longitudinal do aço é $E_{S}=210 \mathrm{GPa}$.

Para realizar o dimensionamento de uma estrutura de concreto armado, pode-se adotar uma simplificação de uma seção retangular com armadura simples, sujeita a um momento fletor de cálculo, resultando no diagrama retangular mostrado na Figura 6.

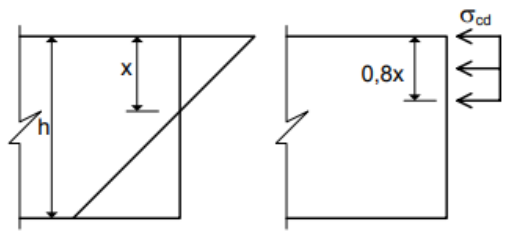

Figura 6: Diagrama retangular para o concreto. Fonte: Araújo (2014a) [3].

A adoção deste diagrama facilita as equações de dimensionamento, fornecendo resultados harmonizáveis com o diagrama parábola-retângulo. A incógnita $x$ representa a posição da linha neutra medida a partir da borda comprimida e considera-se que essa borda comprimida está sujeita a uma tensão constante mostrada na Equação 4, até uma profundidade de 0,8x.

$$
\sigma_{c d}=0,85 f_{c d}
$$

Ao empregar as duas equações de equilíbrio, pode-se realizar o dimensionamento com armadura simples, obtendo assim a Equação 5 e Equação 6.

$$
\begin{gathered}
\xi=1,25(1-\sqrt{1-2 \mu}) \\
A_{s}=0,8 \cdot \xi \cdot b \cdot d \cdot \frac{\sigma_{c d}}{f_{y d}}
\end{gathered}
$$

Onde o $b$ é a largura da seção transversal, $d$ é a altura da seção transversal, e o momento reduzido é dado pela Equação 7. 


$$
\mu=\frac{M_{d}}{b d^{2} \sigma_{c d}}
$$

Utiliza-se essa equação quando $\xi \leq \xi_{\text {lim }}$, onde $\xi_{\text {lim }}$ é um valor limite para a profundidade da linha neutra configurando uma ruptura dúctil.

\subsection{SOFTWARE VISUAL STUDIO}

Segundo o site da Microsoft, o Visual Studio é uma plataforma para desenvolvimento de programação independentemente da linguagem utilizada, seja ela C\#/VB ou C++, JavaScript ou Python.

Pode ser entendida como um software editor de textos que prove aos usuários a possibilidade de redigirem códigos em determinada linguagem computacional, que posteriormente serão assimiladas como comandos para o computador.

Com o software é possível o desenvolvimento de vários tipos de aplicativos e, independentemente da linguagem utilizada, ele é capaz de mostrar os atalhos disponíveis para os comandos, além do preenchimento automático, o que facilita no desenvolvimento e construção do código.

A utilização do Visual Basic se torna interessante por alguns motivos, com a possibilidade de depurar o código que foi desenvolvido, que nada mais é que verificar os erros de sintaxe existentes no código ou na lógica dos comandos do programa.

Ao depurar o código, o software mostra uma lista com os erros existentes, onde é possível identificar em qual a linha se encontra algum erro existente, tanto de sintaxe quanto de lógica. Além disso, dependendo da linguagem utilizada pelo usuário, o programa também exibe opções que podem ser utilizadas para soluções de alguns erros.

Após o termino do desenvolvimento do código, dependendo de sua finalidade, é possível trabalhar em uma interface gráfica visual para os possíveis usuários do programa, e assim gerar um arquivo executável, onde são inseridos parâmetros necessários para a execução de um código, e também obter os resultados gerados pelo programa.

\section{METODOLOGIA}

Primeiramente foi elaborado um estudo teórico sobre as etapas de dimensionamento de lajes de concreto armado, assim como os esforços a que se submetem. Além das lajes de concreto armado, foi também desenvolvido um estudo em algoritmos e na linguagem de programação proposta.

O desenvolvimento do programa foi feito através de linguagem de programação Visual Basic, e está limitado ao estudo e desenvolvimento de uma ferramenta computacional que faça o dimensionamento de lajes de concreto armado, considerando apenas lajes maciças com geometria retangular e carregamento composto unicamente por cargas uniformemente distribuídas.

Esse carregamento pode ser inserido pelo usuário, de acordo com a estrutura desejada e também para a devida utilização a qual será destinada, assim como os parâmetros inicias que irão definir a configuração da laje isolada e os momentos que nela serão aplicados.

No programa desenvolvido, também é possível o usuário escolher qual a opção de apoio deseja aplicar a estrutura a ser dimensionada. A escolha será feita por cada bordo da laje, podendo escolher se o bordo estará simplesmente apoiado, engastada ou livre.

Com os valores de momentos máximos gerados pelo programa é feita uma verificação, comparando esses valores do programa com valores obtidos através das expansões em série de Fourier, utilizando a solução de Navier.

Para o desenvolvimento do programa, se construiu um algoritmo para o dimensionamento das lajes conforme descrito ao longo deste texto e se alimentando o código à medida que foi preciso. Como apresentar o algoritmo em si, poderia não trazer certa clareza para algum leitor leigo do assunto, no decorrer deste capítulo, será apresentado como o programa se comporta no cálculo de lajes maciças em concreto armado, pela forma como pode ser utilizado, demostrando suas principais funções e características. O software em questão, no qual se atribuiu o nome de ProgLaje, 
foi completamente desenvolvido no Visual Studio 2017, devido a simplicidade na manipulação dos dados e na clareza da exposição dos resultados.

A interface geral do programa está divido em abas, que constituem as etapas necessárias para o cálculo das armaduras positivas de uma laje, sendo:
a) Dados da Laje;
b) Carregamento;
c) Esforços;
d) Armadura Positiva;
e) Relatório.

Todos esses itens serão descritos nos itens a seguir.

\subsection{DADOS DE ENTRADA}

Ao se inicializar o programa, a janela que será aberta é como mostrada na Figura 7.

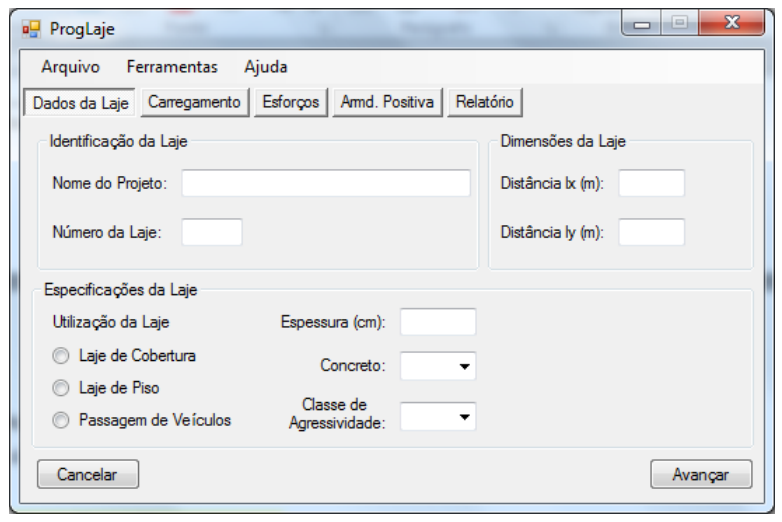

Figura 7: Aba: Dados da Laje. Fonte: autor.

Esta aba corresponde à primeira etapa do projeto, e deve ser totalmente preenchida para dar continuidade às próximas etapas do programa. O primeiro grupo de informações, denominado "Identificação da Laje", tem o princípio de facilitar e organizar a saída de dados. O segundo grupo, denominado "Dimensões da Laje", é onde deve ser inserido os valores para os vãos efetivos da laje, simplificadamente adotados como a distância entre os centros de apoio, e dependendo a da relação entre esses vãos, as lajes serão classificadas como: armada em uma direção ou armada em duas direções. Já o terceiro grupo, denominado "Especificações da Laje", são definidas as propriedades dos materiais, assim como o tipo de ambiente no qual será utilizada.

\subsection{CARREGAMENTO}

Na aba Carregamento, são definidas as cargas atuantes na laje como mostrada na Figura 8, onde é feito o cálculo das cargas, sendo divididas em três grupos de informações. O primeiro grupo, denominado "Tipo de Ambiente", que define a parcela das cargas acidentais de acordo com a NBR 6120:1980 [6]. Para o segundo grupo, denominado "Revestimento", calcula o revestimento escolhido, juntamente com o peso próprio da laje, sendo a parcela de cargas permanentes. Há ainda um terceiro grupo, denominado "Outros Carregamentos", que serve para eventuais carregamentos não listados anteriormente. 


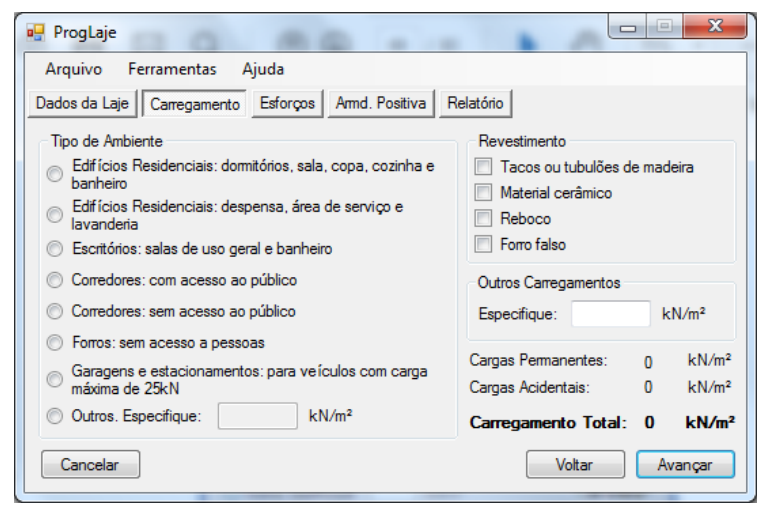

Figura 8: Aba: Carregamento. Fonte: autor.

\subsection{ESFORÇOS SOLICITANTES}

Nesta aba, são apresentados os esforços solicitantes na laje, como demonstrado na Figura 9.

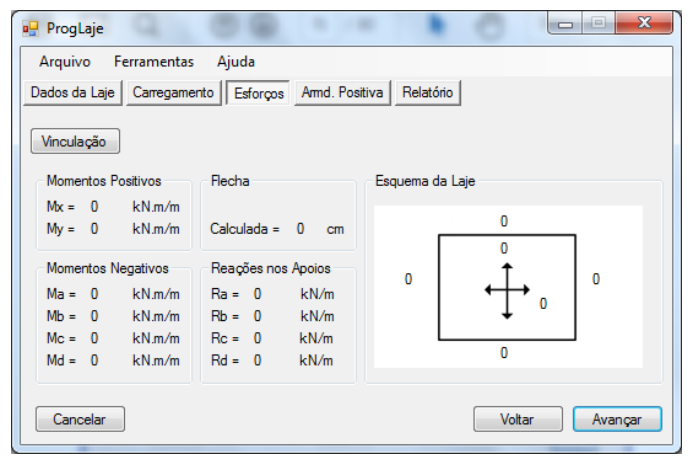

Figura 9: Aba: Esforços. Fonte: autor.

Porém, antes de obter os valores dos esforços solicitantes, é necessário escolher a vinculação na qual a laje está submetida. Ao clicar no botão "Vinculação", será aberta uma nova janela para que se escolham as condições de contorno da laje, como mostrado na Figura 10.

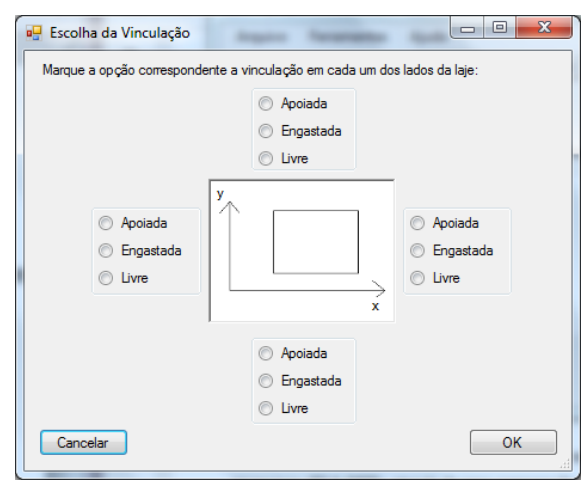

Figura 10: Aba: Escolha da Vinculação. Fonte: autor.

\subsection{ARMADURA POSITIVA}

Na aba Armd. Postivia é apresentada a área de aço necessária nos vãos, colocados junto à face inferior da laje, para as respectivas direções x e y, como mostrada na Figura 11. 


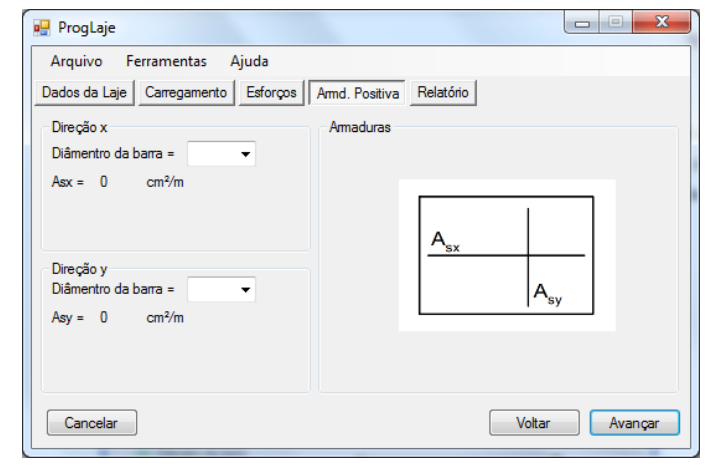

Figura 11: Aba: Armadura Positiva. Fonte: autor.

Para a definição da armadura em cada direção, o usuário deve escolher o diâmetro do aço na qual deseja utilizar, e após isto, automaticamente é calculado o espaçamento entre as barras, assim como o número de barras necessárias e seu respectivo comprimento.

\section{RESULTADOS}

Para verificação dos valores obtidos, foi feita uma comparação com lajes de variadas dimensões e condições de contorno simuladas pelo programa, comparando com as expansões em série de Fourier, utilizando a solução de Navier, apresentadas no livro Theories and Application of Plates Analysis (2004) [7], sendo elas:

$$
P_{m n}=\frac{16 p}{\pi^{2} m n}
$$

Onde $P_{m n}$ é o coeficiente de carga para cargas uniformemente distribuídas dadas pela Equação 8 , sendo o valor de p o carregamento aplicado na laje. Obtêm-se também o coeficiente $W_{m n}$ pela Equação 9.

$$
W_{m n}=\frac{P_{m n}}{D \pi^{4}\left[\left(\frac{m^{2}}{a^{2}}\right)+\left(\frac{n^{2}}{b^{2}}\right)\right]^{2}}
$$

Obtido este coeficiente, é possível obter os esforços solicitantes por simples diferenciação dos momentos e da flecha, através da Equação 10 e Equação 11.

$$
\begin{aligned}
& M_{x}=\pi^{2} D \sum_{m=1}^{\infty} \sum_{n=1}^{\infty}\left[\left(\frac{m}{a}\right)^{2}+v\left(\frac{n}{b}\right)^{2}\right] W_{m n} \sin \frac{m \pi x}{a} \sin \frac{m \pi y}{b} \\
& M_{y}=\pi^{2} D \sum_{m=1}^{\infty} \sum_{n=1}^{\infty}\left[\left(\frac{n}{b}\right)^{2}+v\left(\frac{m}{a}\right)^{2}\right] W_{m n} \sin \frac{m \pi x}{a} \sin \frac{m \pi y}{b}
\end{aligned}
$$

Com isso, obtêm-se os valores dos esforços solicitantes através da solução de Navier. A verificação apresentada foi baseada em dados comumente empregados num edifício residencial: para ambiente sala/dormitórios, com classe de agressividade ambiental II, utilizando uma espessura de laje de $10 \mathrm{~cm}$, executada com um fck de $30 \mathrm{Mpa}$. Para o revestimento foi considerada uma carga de $1 \mathrm{kN} / \mathrm{m}^{2}$, resultando em uma carga de $5 \mathrm{kN} / \mathrm{m}^{2}$. Nos resultados do programa, apresentou-se os resultados para bordos simplesmente apoiados, engastados em uma direção e também livre em uma direção, de forma a demonstrar o funcionamento do mesmo. Para a verificação, utilizou-se os bordos simplesmente apoiados, como irá ser demonstrado abaixo.

$\mathrm{Na}$ Tabela 1 são mostrados os valores encontrados pelo programa ProgLaje. 
Tabela 1: Resultados encontrados pelo programa ProgLaje em kNm. Fonte: autor.

\begin{tabular}{ccccccccc}
\hline & & \multicolumn{9}{c}{ ProgLaje } \\
\cline { 3 - 9 } & & Simpl. Apoiada & \multicolumn{2}{c}{ Engastada 1 direção } & \multicolumn{2}{c}{$\begin{array}{c}\text { Livre 1 } \\
\text { direção }\end{array}$} \\
\hline $\begin{array}{c}\text { Direção } \\
\mathbf{x}(\mathbf{m})\end{array}$ & $\begin{array}{c}\text { Direção } \\
\mathbf{y}(\mathbf{m})\end{array}$ & $\mathbf{M x}$ & $\mathbf{M y}$ & $\mathbf{M x}$ & $\mathbf{M y}$ & $\mathbf{M e}$ & $\mathbf{M x}$ & $\mathbf{M y}$ \\
\hline 3 & 3 & 1,98 & 1,98 & 1,39 & 1,61 & $-3,75$ & 3,50 & 1,63 \\
3 & 4 & 3,08 & 1,99 & 2,47 & 1,86 & $-4,75$ & 4,28 & 1,59 \\
3 & 5 & 3,90 & 1,83 & 3,41 & 1,84 & $-5,22$ & 4,75 & 1,49 \\
4 & 5 & 5,03 & 3,58 & 3,93 & 3,26 & $-8,12$ & 7,31 & 2,86 \\
5 & 5 & 5,50 & 5,51 & 3,86 & 4,48 & $-10,43$ & 9,71 & 4,54 \\
\hline
\end{tabular}

Na solução de Navier são utilizados como parâmetros de entrada para as equações a espessura da laje de $10 \mathrm{~cm}$, a carga total de $5 \mathrm{kN} / \mathrm{m}^{2}$, o coeficiente de Poisson do concreto de 0,2 e o módulo de elasticidade com o valor de $28518 \mathrm{GPa}$.

Na Tabela 2 são mostrados os valores encontrados através das expansões em série de Fourier, adotando a solução de Navier.

Tabela 2: Resultados encontrados pela solução de Navier em kNm. Fonte: autor.

\begin{tabular}{cccc}
\hline & & \multicolumn{2}{c}{ Solução de Navier } \\
\cline { 2 - 4 } & & \multicolumn{2}{c}{ Simplesmente Apoiada } \\
\hline $\begin{array}{c}\text { Direção x } \\
(\mathbf{m})\end{array}$ & $\begin{array}{c}\text { Direção } \mathbf{y} \\
(\mathbf{m})\end{array}$ & $\mathbf{M x}$ & $\mathbf{M y}$ \\
\hline 3 & 3 & 1,992 & 1,992 \\
3 & 4 & 3,079 & 1,992 \\
3 & 5 & 3,915 & 1,837 \\
4 & 5 & 5,027 & 3,581 \\
5 & 5 & 5,535 & 5,535 \\
\hline
\end{tabular}

Na Tabela 3, pode-se verificar a que o programa é eficiente, visto que as diferenças entre os métodos ficaram da ordem de 0,03 a $0,60 \%$ entre os resultados do programa desenvolvido e a solução analítica de Navier.

Tabela 3: Diferenças encontradas entre a solução de Navier e o apresentado no ProgLajes, em \%. Fonte: autor.

\begin{tabular}{cccc}
\hline & & \multicolumn{2}{c}{ Simplesmente Apoiada } \\
\hline $\begin{array}{c}\text { Direção x } \\
(\mathbf{m})\end{array}$ & $\begin{array}{c}\text { Direção y } \\
(\mathbf{m})\end{array}$ & $\mathbf{M x}(\boldsymbol{\%})$ & $\mathbf{M y}(\boldsymbol{\%})$ \\
\hline 3 & 3 & 0,60 & 0,60 \\
3 & 4 & 0,03 & 0,10 \\
3 & 5 & 0,38 & 0,38 \\
4 & 5 & 0,06 & 0,03 \\
5 & 5 & 0,63 & 0,45 \\
\hline
\end{tabular}

\section{CONCLUSÃO}

O programa desenvolvido permite definir a vinculação da laje, as características do material, compor as cargas atuantes na laje e, por fim, calcular os esforços. Na elaboração do programa, tomou-se o cuidado em respeitar os critérios cabíveis estabelecidos tanto pela NBR 6118:2014 quanto pela NBR 6120: 1980.

Por meio de uma interface gráfica intuitiva, o programa visa simplicidade e praticidade na sua utilização, uma vez que todas as etapas foram elaboradas no intuído de calcular os esforços de uma laje de forma rápida e com fácil leitura dos resultados. 
De uma maneira geral, tendo-se em vista os resultados obtidos após a verificação, fica claro que o programa desenvolvido pode ser eficientemente adotado por acadêmicos do curso de Engenharia Civil, bem como para profissionais da área em questão. Todavia, é altamente recomendável que o usuário tenha ciência de todas as etapas do cálculo.

Como proposta de trabalhos futuros, pode-se ampliar o programa para o cálculo das armaduras das lajes de concreto armado e suas verificações.

\section{AGRADECIMENTOS}

Agradecemos aqueles que, de alguma forma, contribuíram para que este artigo fosse realizado.

\section{REFERÊNCIAS BIBLIOGRÁFICAS}

1. Kimura A. Informática aplicada em estruturas de concreto armado: cálculos de edifícios com uso de sistemas computacionais. São Paulo: Pini; 2007. 624 p.

2. Associação Brasileira de Normas Técnicas. NBR 6118 - Projetos de estruturas de concreto armado; 2014.

3. Araújo JM. Curso de concreto armado. V2. $4^{\text {a }}$ edição. Rio Grande: Editora Dunas; 2014. 421 p.

4. Cunha AJP; Souza VC. Lajes em concreto armado e protendido. $2^{\mathrm{a}}$ edição. Niterói: Ed. Universidade Federal Fluminense; 1998. 580 p.

5. Araújo JM. Curso de concreto armado. V1. $4^{\mathrm{a}}$ edição. Rio Grande: Editora Dunas; 2014. 303 p.

6. Associação Brasileira de Normas Técnicas. NBR 6120 - Cargas para o cálculo de estruturas de edificações; 1980.

7. Szilard R. Theories and Application of Plates Analysis: Classical, Numerical and Engineering Methods. New Jersey: Ed. John Wiley \& Sons, Inc; 2004. 1024 p. 